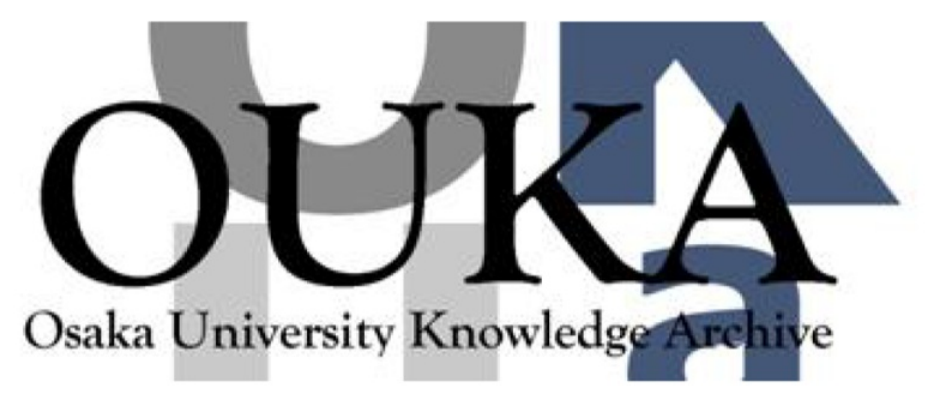

\begin{tabular}{|c|l|}
\hline Title & $\begin{array}{l}\text { Elimination of bandwidth effect in attenuation } \\
\text { measurement with picosecond ultrasonics }\end{array}$ \\
\hline Author(s) & $\begin{array}{l}\text { Maehara, Atsushi; Nakamura, Nobutomo; 0gi, } \\
\text { Hirotsugu et al. }\end{array}$ \\
\hline Citation & $\begin{array}{l}\text { Japanese Journal of Applied Physics. 53(8) } \\
\text { p. 086602 }\end{array}$ \\
\hline Issue Date & $2014-07-28$ \\
\hline oaire:version & AM \\
\hline URL & https://hdl. handle. net/11094/84484 \\
\hline rights & \\
\hline Note & \\
\hline
\end{tabular}

Osaka University Knowledge Archive : OUKA

https://ir. Library. osaka-u. ac. jp/

Osaka University 


\title{
Elimination of bandwidth effect in attenuation measurement with picosecond ultrasonics
}

\author{
Atsushi Maehara, Nobutomo Nakamura*, Hirotsugu Ogi, and Masahiko Hirao \\ Graduate School of Engineering Science, Osaka University, Toyonaka, Osaka 560-8531, Japan
}

\begin{abstract}
We study the broadening effect of probing pulse light on the apparent attenuation of the Brillouin oscillation measured with picosecond ultrasonics. We observe experimentally that the attenuation of the Brillouin oscillation is sensitive to the bandwidth, and the apparent attenuation coefficient increases as the bandwidth increases, being far from the intrinsic attenuation coefficient. Theoretical calculation is performed to reconstruct the observed oscillations, and it is confirmed that there are several factors affecting the apparent attenuation in addition to the bandwidth. We finally propose equations that deduce the contribution of the broadening to the apparent attenuation of the Brillouin oscillation.
\end{abstract}

\section{Introduction}

Acoustic attenuation originates from several factors such as scattering, dislocation damping, and phonon-phonon interactions. ${ }^{1)}$ Attenuation measurement has been, therefore, a powerful method for characterizing materials. Attenuation by scattering is applicable to the grain-size measurements of polycrystalline metals. ${ }^{2,3)}$ The change in the acoustic attenuation with the evolution of the dislocation structure is promising for use in remaining-life estimation of fatigued materials. ${ }^{4,5)}$ In the development of acoustic filters and sensors, the attenuation coefficient is a key factor; greater acoustic attenuation of the constituent materials lowers the quality factor of the devices.

In the context of increasing importance of high-frequency acoustic devices, acoustic attenuation at high frequencies is attracting attention. Among several photoacoustic techniques, Brillouin oscillation caused by picosecond ultrasonics ${ }^{6,7)}$ allows us to explore acoustic properties of transparent and translucent materials. It employs acoustic phonons of frequency higher than $100 \mathrm{GHz}$, corresponding to wavelength smaller than $100 \mathrm{~nm}$, and it has been applied to the measurement of elastic stiffness. ${ }^{8-10)}$ This technique excites and detects acoustic phonons without contact with the specimen and thus allows a measurement without the influence of the contact between the transducer and

${ }^{*}$ E-mail: nobutomo@me.es.osaka-u.ac.jp 
specimen. Therefore, it has also been used for the attenuation measurement. ${ }^{11-14)}$ However, when the attenuation coefficient is determined from the damping of the Brillouin oscillation, there is a concern. Brillouin oscillation is detected through the reflectivity change in the probe light reflected from a specimen in which acoustic phonons are propagating; the probe light is reflected at both the specimen surface and the acoustic pulse; the latter occurs by backward diffraction. Damping of the observed reflectivity change then reflects not only the attenuation of acoustic phonons but also the interference among components of probe light of different wavelengths diffracted by the broad-band acoustic pulse. Therefore, the attenuation coefficient measured with a wider bandwidth of the probe light pulse becomes larger than the actual attenuation coefficient of a specimen because of the interference. This contribution should be considered to obtain a reliable value of the attenuation coefficient, but it has not been considered in previous studies. We evaluate the contribution of the bandwidth to the attenuation measurement through experiments. Reconstruction of the reflectivity change by theoretical calculations is then performed, and a significant effect of bandwidth variation on the attenuation measurement is shown. We propose a simple and useful method for estimating the bandwidth effect after comprehensive analysis.

\section{Experimental procedure}

Brillouin oscillation is observed in transparent materials using picosecond ultrasonics. A metallic thin film is deposited on a specimen surface as a sound source. When a pump light irradiates the metallic film, an acoustic pulse is generated in the film by the instantaneous thermal expansion-shrinkage process, and propagates in the normal direction to the surface. When a probe light irradiates the specimen, a part of it transmits into the specimen and is diffracted backward by the acoustic pulse because of a local change in the dielectric constant in accordance with the photoelastic effect. The diffracted light then interferes with the light reflected at the surface, and an oscillating intensity change is induced in the interfered light while changing the delay time, reflecting the propagation of the acoustic pulse. This oscillation is called the Brillouin oscillation. Theoretical models for the reflectivity change measured using the picosecond ultrasonics have been established in the previous studies. ${ }^{7,8)}$

In this study, two optical systems with different probe light bandwidths are developed for measuring Brillouin oscillation. In both systems, two Ti-sapphire pulse lasers are used for the pump and probe lights, and the fundamental design of the optics are the 
same, although there are minor differences. Figure 1 shows the optics of one of them. One of the two Ti-sapphire pulse lasers, the so-called slave (Coherent Vitara), is synchronized with another one, the so-called master (Coherent Mira), by the synchronizing system (Coherent SynchroLock-AP) with a timing jitter less than 250 fs. For the synchronization, the lights reflected at beam samplers (BSmps) are used. The pump light with the center wavelength of $800 \mathrm{~nm}$, the repetition rate of $80 \mathrm{MHz}$, and the pulse width of $123 \mathrm{fs}$ is modulated for lock-in detection by passing through the acousto-optic modulator (AOM). Primary diffracted light is reflected at a dichroic mirror (DM) to irradiate the specimen surface perpendicularly. The probe light with the center wavelength of $800 \mathrm{~nm}$, the repetition rate of $80 \mathrm{MHz}$, and the pulse width of 35 fs passes through the second-harmonic-generation (SHG) crystal to generate second-harmonic light with the center wavelength of $400 \mathrm{~nm}$. Half of the light is used for the reference light of the balanced detector and the other half passes through the DM to irradiate the specimen with the same light axis as that of the pump light. The reflected light is detected by the balanced detector. By a change in the length of the delay line by moving the corner reflector, sub-picosecond time resolution is achieved. The time range changed with the delay line is about 1300 ps. The power of the lights is changed by combining the half-wavelength plate $(\lambda / 2)$ and polarized beam splitter (PBS). The probe beam diameter is about $3 \mathrm{~mm}$. The magnification of the objective lens is twenty and its numerical aperture is 0.35 . The optics of another system is described elsewhere. ${ }^{10)}$ Details of the typical pump-probe technique appear elsewhere. ${ }^{15)}$ The bandwidth $d$, full width at half maximum (FWHM), of the probe light is different for each system, and it is 2.3 and $7.1 \mathrm{~nm}$. The intensity spectrum of the probe light measured using a spectrometer with a resolution of $0.94 \mathrm{~nm}$ is shown in Fig. 2. The values of the center wavelength $\lambda_{0}$ differ slightly from each other: $399.9 \mathrm{~nm}$ for $d=2.3 \mathrm{~nm}$ and $400.7 \mathrm{~nm}$ for $d=7.1 \mathrm{~nm}$.

In the experiment, monocrystalline $\mathrm{SrTiO}_{3}$ was adopted for the negligible light absorption around $400 \mathrm{~nm}^{16)}$ to eliminate the optical attenuation factor. The specimen size is $4.7 \times 3.7 \times 0.5 \mathrm{~mm}^{3}$. The wavenumber $k$ dependence of the refractive index $n(k)$ was measured by ellipsometry measurement. Pt film of $18 \mathrm{~nm}$ thick was deposited on a (100) surface, and the acoustic pulse propagating in the $\langle 100\rangle$ direction was investigated at room temperature. The film thickness was determined by X-ray reflectivity measurement. $\left.{ }^{17}\right)$ The power of both the pump and probe lights was $20 \mathrm{~mW}(0.25 \mathrm{~nJ}$ per pulse). Figures 3(a) and 3(b) show the measured intensity change for the two cases. The background signal originating from heat dispersion has been extracted. For $d=2.3 \mathrm{~nm}$, 
Brillouin oscillation lasted for $600 \mathrm{ps,} \mathrm{while} \mathrm{for} d=7.1 \mathrm{~nm}$ the oscillation disappeared at around 300 ps. It is obvious that apparent attenuation behaviors differ from each other, indicating that the acoustic attenuation in the specimen cannot be simply evaluated from the attenuation in the Brillouin oscillation. Hereafter, the acoustic attenuation in a specimen is called intrinsic attenuation, and the attenuation in the Brillouin oscillation is called apparent attenuation.

\section{Brillouin oscillation with broadening of probe light}

Referring to the previous studies, we calculate the intensity change of the reflected probe light considering the bandwidth effect. In the experiment, Pt film was deposited on the (100) surface of $\mathrm{SrTiO}_{3}$ as a sound source, but the propagation of an acoustic pulse in the film is neglected in the following calculation. When the probe light is reflected with reflectivity coefficient $r(k)$, the electric field of the reflected probe light is expressed as

$$
E_{r}=\int_{-\infty}^{\infty} E(k) r(k) e^{-\mathrm{i} k(c t+z)} \mathrm{d} k
$$

Here, $E(k)$ is the amplitude of the electric field of the incident probe light at $k$, and $c$ is the velocity of light in vacuum. We take the $z$ axis parallel to the propagation direction of the acoustic pulse to be normal to the surface. The origin is the specimen's surface, and the positive direction of the axis is toward the specimen. The reflectivity coefficient from a vacuum to the specimen and that of backward diffraction by an acoustic pulse in the specimen are given by

$$
\begin{gathered}
r_{01}(k)=\frac{\sqrt{\epsilon_{0}}-\sqrt{\epsilon(k)}}{\sqrt{\epsilon_{0}}+\sqrt{\epsilon(k)}}, \\
\Delta r_{1}(k, t)=\int_{0}^{\infty} F(k) e^{-2 \mathrm{in} k z} p \eta(t, z) \mathrm{d} z,
\end{gathered}
$$

respectively. $\epsilon_{0}$ and $\epsilon(k)$ denote the dielectric constant in vacuum and that in the specimen, respectively, and $n$ is the refractive index, $n=\sqrt{\epsilon(k) / \epsilon_{0}}$. In $\Delta r_{1}(k, t)$, transmission of the light from a vacuum to a specimen, the backward diffraction, and transmission from the specimen to the vacuum are taken into account. $p=(\partial \epsilon(k) / \partial \eta) / \epsilon_{0}$ is the piezo-optic constant. The sensitivity weight $F$ and the strain field $\eta$ are expressed by

$$
\begin{gathered}
F(k)=-\frac{\mathrm{i} k}{2 n}\left(1-r_{01}^{2}(k)\right), \\
\eta(t, z)=-\eta_{0} e^{-\alpha t} \exp \left(-\frac{|z-v t|}{\zeta}\right) \operatorname{sgn}(z-v t) .
\end{gathered}
$$


$\eta_{0}$ is a positive constant. $\zeta$ is the spatial width of the acoustic pulse. $v$ and $\alpha$ are the acoustic velocity and the time-domain attenuation coefficient of the acoustic pulse, respectively. The intensity of the probe light is proportional to the square of $E_{r}$, and

$$
I \approx\left|E_{r}\right|^{2}=\int_{-\infty}^{\infty} \int_{-\infty}^{\infty} E(k) E\left(k^{\prime}\right) r(k) r^{*}\left(k^{\prime}\right) e^{-\mathrm{i}\left(k-k^{\prime}\right)(v t-z)} \mathrm{d} k \mathrm{~d} k^{\prime} .
$$

$r^{*}$ is the conjugate of $r$. Considering that time average of the intensity is observed by a detector, cross terms, $e^{-\mathrm{i}\left(k-k^{\prime}\right)(v t-z)}\left(k \neq k^{\prime}\right)$, becomes negligible,

$$
I \approx \int_{-\infty}^{\infty} E^{2}(k)|r(k)|^{2} \mathrm{~d} k
$$

and the intensity change due to the acoustic pulse propagating in a specimen is expressed as

$$
\Delta I(t) \approx \int_{-\infty}^{\infty} E^{2}(k)\left|r_{01}(k)+\Delta r_{1}(k, t)\right|^{2} \mathrm{~d} k-\int_{-\infty}^{\infty} E^{2}(k)\left|r_{01}(k)\right|^{2} \mathrm{~d} k .
$$

\section{Results and discussion}

Our aim is the determination of $\alpha$. We determine $\alpha$ by fitting Eq. (8) to the measured intensity changes. Before the fitting, $v$ is determined from the center wavelength of the probe light, $n$ at the center wavelength, and the oscillation frequency observed in the fast-Fourier transform spectrum of the measured intensity change, assuming monochromatic probe light. ${ }^{8)}$ The intensity profiles of the probe light used in the calculation are plotted in Fig. 2. They were obtained by fitting the Gaussian function to the measured optical spectra, and interference between them was considered. $\zeta$ is assumed to be the same as the thickness of Pt film, $18 \mathrm{~nm}$. At this time, $\alpha$, the product of $p$ and $\eta_{0}$, and the factor of proportionality between left and right sides of Eq. (8) are unknown parameters. $\alpha$ was determined by fitting the intensity change. The fitted curve is shown in the second row of Figs. 3(a) and 3(b), and it is compared with the measured curve in Fig. 3(c). The fitted intensity change shows good agreement with the measured one, and $\alpha$ is 2.8 and $2.1 \mathrm{~ns}^{-1}$ for $d=2.3$ and $7.1 \mathrm{~nm}$, respectively. These are close to $\alpha=2.0$ $\mathrm{ns}^{-1}$ deduced from the reported decay time. ${ }^{18)}$

To evaluate the contribution of bandwidth and acoustic attenuation to the apparent attenuation, intensity change was calculated for two cases: (i) considering the acoustic attenuation and excluding the effect of bandwidth assuming a monochromatic probe light, (ii) considering the effect of bandwidth and no acoustic attenuation $(\alpha=0)$. The calculated intensity change is shown in Figs. 3(a) and 3(b). For $d=2.3 \mathrm{~nm}$, measured intensity change shows a similar attenuation behavior to the one calculated with only the 
acoustic attenuation. In the case of $d=7.1 \mathrm{~nm}$, effect of bandwidth becomes a dominant factor in the apparent attenuation. These results indicate that a narrow bandwidth of probe light is important for accurate attenuation measurement, and the bandwidth of $7.1 \mathrm{~nm}$ around $400 \mathrm{~nm}$ is not sufficiently narrow for $\mathrm{SrTiO}_{3}$.

In the above discussion, we demonstrated that the proposed model reproduces the measured Brillouin oscillation, and the fitting of the intensity change can deduce the attenuation coefficient. We here consider another method for estimating the contribution of bandwidth without fitting. To find regularity between the apparent attenuation and intrinsic attenuation, the apparent attenuation coefficient $\alpha_{a}$ is calculated with different acoustic velocity $v$, refractive index $n$, center wavelength of the probe light $\lambda_{0}$, and wavelength dependence of the refractive index $n^{\prime}$ using Eq. (8). The wavelength dependence of the refractive index is defined by the slope in $n(\lambda)=n^{\prime}\left[\left(\lambda-\lambda_{0}\right) / \lambda_{0}\right]+n\left(\lambda_{0}\right)$. In the calculations, the Gaussian function was assumed for the wavelength dependence of the intensity profile of the probe light seen in Fig. 2, and the intensities at twenty-one wavelengths were used. Apparent attenuation coefficient $\alpha_{a}$ is determined from the FWHM of the FFT spectrum of the intensity change as shown in Fig. $4(\mathrm{a}), \alpha_{a}=\pi f \times \mathrm{FWHM} / \sqrt{3}$, in which the window function is not used. In the calculations, input parameters are $v=8000 \mathrm{~m} / \mathrm{s}, n=3, \lambda_{0}=400 \mathrm{~nm}$, and $n^{\prime}=0$; one of the parameters was changed [Figs. 4(b)-(e)]. The film thickness of the sound source, corresponding to the pulse width, is assumed to be $10 \mathrm{~nm}$. In each figure, the $d$ dependence of $\alpha_{a}$ is calculated at $\alpha=0,2$, and $20 \mathrm{~ns}^{-1}$. In the calculation results, as $d$ increases, $\alpha_{a}$ increases, and increment ratio varies with the value of the parameters. The bandwidth dependence of apparent attenuation appear to be complicated. However, $\alpha_{a}$ appears on a specific curve by normalizing the vertical axis by $\alpha^{\prime}=\alpha_{a} / \alpha$ and the horizontal axis by $d_{1}=d v n\left(1-n^{\prime} / n\right) /\left(\lambda_{0}^{2} \alpha\right)$ or $d_{2}=d v n\left(1-n^{\prime} / n\right) /\left(\lambda_{0}^{2} \alpha_{a}\right)$, as seen in Figs. $4(\mathrm{f})$ and $4(\mathrm{~g})$, respectively. $d_{1}$ and $d_{2}$ denote the relative bandwidth to the center wavelength, which were deduced by considering that the relative bandwidth increases with decreasing center wavelength and increasing absolute value of $n^{\prime}$. Larger $v$ increases the frequency of the Brillouin oscillation, and the attenuation by the interference becomes greater as if the relative bandwidth increases. In Fig. 4(f), at around $d_{1}=0, \alpha^{\prime}$ gradually increases with $d_{1}$, and it approaches a linear function at larger $d_{1}$. The trend is expressed as

$$
\alpha^{\prime}=0.574+6.407 d_{1}+0.426 e^{-14.935 d_{1}} .
$$

In Fig. $4(\mathrm{~g})$, as $d_{2}$ increases, $\alpha^{\prime}$ gradually increases, and at around $d_{2}=0.15, \alpha^{\prime}$ increases 
rapidly. An approximation curve is obtained as

$$
\alpha^{\prime}=\frac{3.74 \times 10^{-3}}{\left(d_{2}-0.116\right)^{2}}-\frac{7.60 \times 10^{-7}}{\left(d_{2}-0.116\right)^{4}}+\frac{2.54 \times 10^{-10}}{\left(d_{2}-0.116\right)^{6}}+0.864
$$

These equations give a comprehensive relationship between bandwidth and apparent attenuation, and allow us to estimate the contribution of the broadening of the probe light. For example, when the approximate value of $\alpha$ of a specimen is known, the bandwidth effect can be estimated before measurement using Eq. (9). In the case of $\mathrm{SiO}_{2}$, using the reported attenuation factor, ${ }^{12)}$ velocity, ${ }^{12)}$ and refractive index, ${ }^{19)} \alpha^{\prime}$ is estimated to be $1.5,2.9$, and 5.2 at $d=2,5$, and $10 \mathrm{~nm}$, respectively, with the probe light of $\lambda_{0}=400 \mathrm{~nm} . \alpha^{\prime}=2.9$ means that the contribution of the bandwidth effect is almost twice as large as the intrinsic attenuation. To make the contribution smaller than $10 \%, d$ must be smaller than $0.7 \mathrm{~nm}$. Thus, the bandwidth effect is estimated in advance using Eq. (9) and known $\alpha$, and the wavelength of the probe light that should be used for a specific specimen is obtained. On the other hand, when $\alpha$ is unknown, Eq. (10) allows us to estimate the contribution of the bandwidth effect after the measurement using $\alpha^{\prime}$ and other parameters. These two equations are helpful for evaluating the contribution of the bandwidth effect.

\section{Conclusions}

Brillouin oscillation in $\mathrm{SrTiO}_{3}$ was investigated using the probe lights with different bandwidths, and faster attenuation of the Brillouin oscillation was observed for the probe light with broader bandwidth. The reconstruction of Brillouin oscillation considering the bandwidth variation allowed us to evaluate its effect on the attenuation of the observed oscillation, and the intrinsic attenuation coefficient was obtained. After comprehensive analysis, equations for determining intrinsic attenuation coefficient from the apparent attenuation coefficient were proposed.

\section{Acknowledgment}

This research was supported by the Innovative Project for Advanced Instruments, Renovation Center of Instruments for Science Education and Technology, Osaka University. 


\section{References}

1) R. Truell, C. Elbaum, and B. B. Chick, Ultrasonic Methods in Solid State Physics (Academic Press, New York, 1969), p. 159.

2) E. P. Papadakis, J. Acoust. Soc. Am. 37, 711 (1965).

3) H. Ogi, M. Hirao, and T. Honda, J. Acoust. Soc. Am. 98, 458 (1995).

4) M. Hirao, H. Ogi, N. Suzuki, and T. Ohtani, Acta Mater. 48, 517 (2000).

5) H. Ogi, Y. Minami, and M. Hirao, J. Appl. Phys. 91, 1849 (2002).

6) C. Thomsen, J. Strait, Z. Vardeny, H. J. Maris, J. Tauc, and J. J. Hauser, Phys. Rev. Lett. 53, 989 (1984).

7) C. Thomsen, H. T. Grahn, H. J. Maris, and J. Tauc, Phys. Rev. B 34, 4129 (1986).

8) H. Ogi, T. Shagawa, N. Nakamura, M. Hirao, H. Odaka, and N. Kihara, Phys. Rev. B 78, 134204 (2008).

9) A. Nagakubo, H. Ogi, H. Sumiya, K. Kusakabe, and M. Hirao, Appl. Phys. Lett. 102, 241909 (2013).

10) K. Tanigaki, H. Ogi, H. Sumiya, K. Kusakabe, N. Nakamura, M. Hirao, and H. Ledbetter, Nat. Commun. 4, 2343 (2013).

11) H. N. Lin, R. J. Stoner, H. J. Maris, and J. Tauc, J. Appl. Phys. 69, 3816 (1991).

12) P. Emery and A. Devos, Appl. Phys. Lett. 89, 191904 (2006).

13) E. Pontecorco, M. Ortolani, D. Polli, M. Ferretti, G. Ruocco, G. Cerullo, and T. Scopigno, Appl. Phys. Lett. 98, 011901 (2011).

14) A. Nagakubo, A. Yamamoto, K. Tanigaki, H. Ogi, N. Nakamura, and M. Hirao, Jpn. J. Appl. Phys. 51, 07GA09 (2012).

15) E. P. Ippen and C. V. Shank, in Ultrashort Light Pulses, ed. S. L. Shapiro (Springer, New York, 1984 ) 2nd de., p. 102.

16) M. Cardona, Phys. Rev. 140, A651 (1965).

17) L. G. Parratt, Phys. Rev. 95, 359 (1954).

18) R. Shayduk, M. Herzog, A. Bojahr, D. Schick, P. Gaal, W. Leitenberger, H. Navirian, M. Sander, J. Goldshteyn, I. Vrejoiu, and M. Bargheer, Phys. Rev. B 87, 184301 (2013).

19) I. H. Malitson, J. Opt. Soc. Am. 55, 1205 (1965). 


\section{Figure captions}

Fig. 1 Schematic illustration of experimental apparatus.

Fig. 2 Intensity profile of the probe lights measured with an optical spectrometer. Solid and dashed curves denote those of bandwidths of 7.1 and $2.3 \mathrm{~nm}$, respectively. Solid circles denote the intensity profile used in the calculation. They were obtained by fitting the Gaussian function to the measured optical spectra.

Fig. 3 Measured and calculated Brillouin oscillation in $\mathrm{SrTiO}_{3}$ with bandwidths of (a) $2.3 \mathrm{~nm}$ and (b) $7.1 \mathrm{~nm}$. Calculation was performed for the three cases of considering both acoustic attenuation and bandwidth (BW) effect, only the acoustic attenuation, and only the BW. (c) Dashed curve denotes the measured intensity change. Solid curve denotes the calculated one with the acoustic attenuation and BW effect taken into consideration.

Fig. 4 Attenuation coefficients calculated for various conditions. (a) Example of calculated intensity change. Inset shows the corresponding FFT spectrum. (b)-(e) Bandwidth dependence of apparent attenuation coefficient with different parameters. In each figure, calculation was performed at $\alpha=0,2$, and $20 \mathrm{~ns}^{-1}$. Solid and open circles denote the calculated values, and they are connected with solid and dashed lines, respectively. (f), (g) Normalized attenuation coefficients appearing in (b)-(e) are plotted, except for $\alpha=0 \mathrm{~ns}^{-1}$. Solid curve denotes the approximation. 


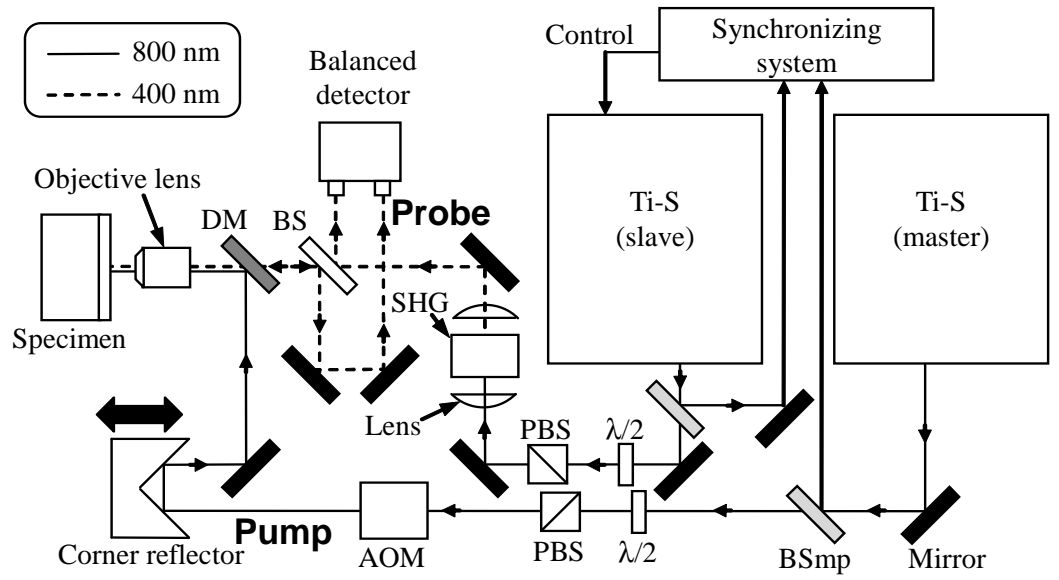

Fig. 1 


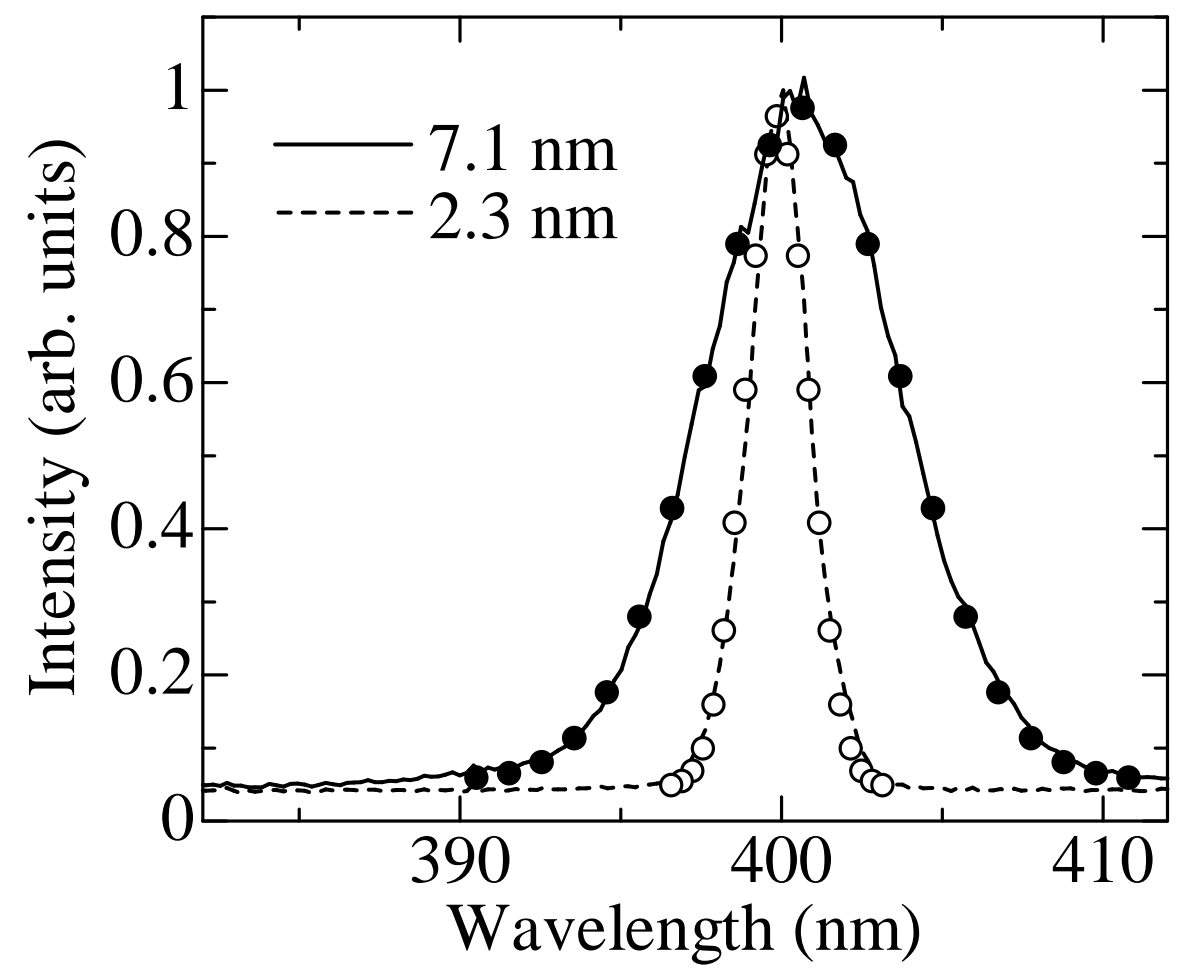

Fig. 2 

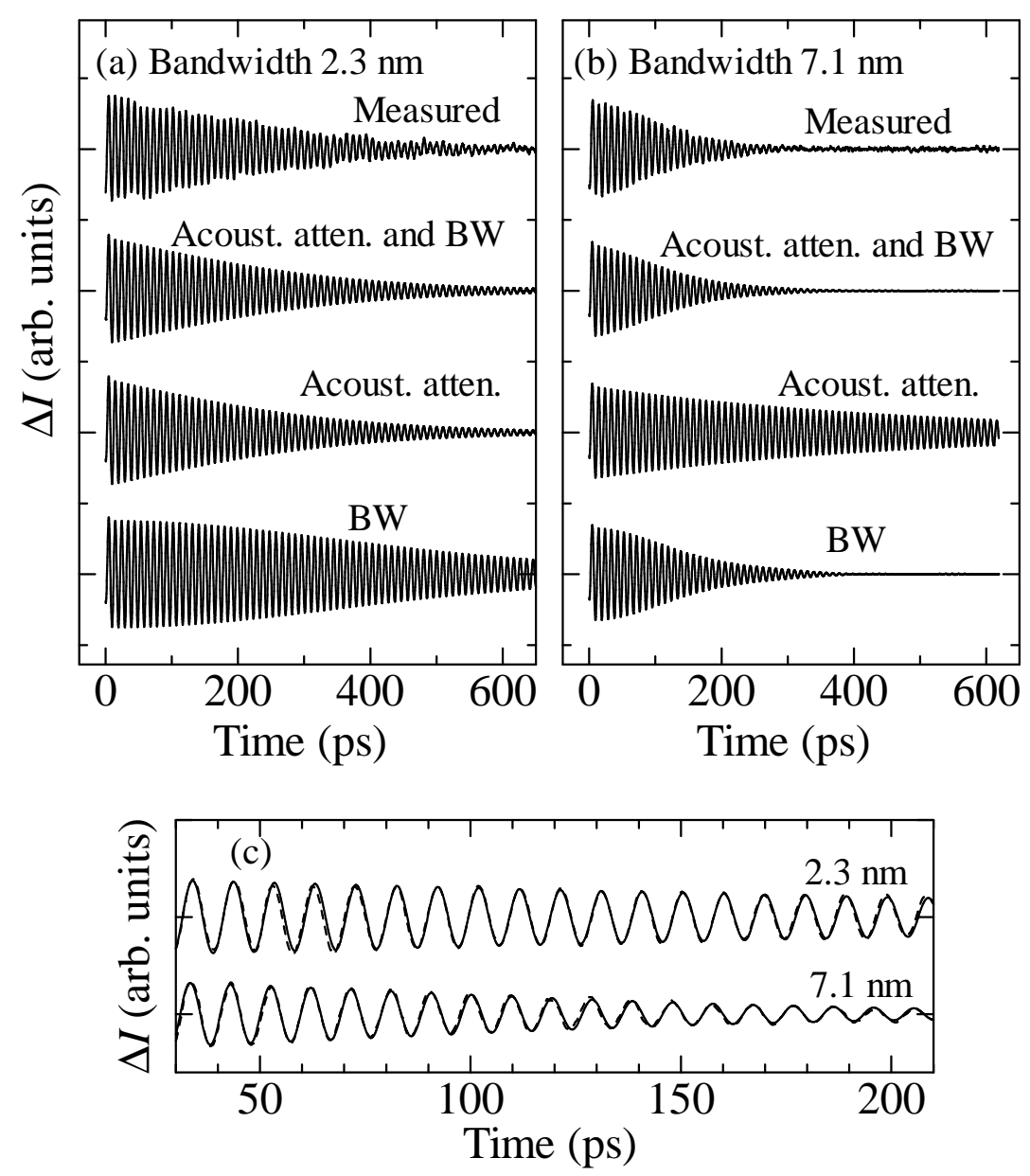

Fig. 3 

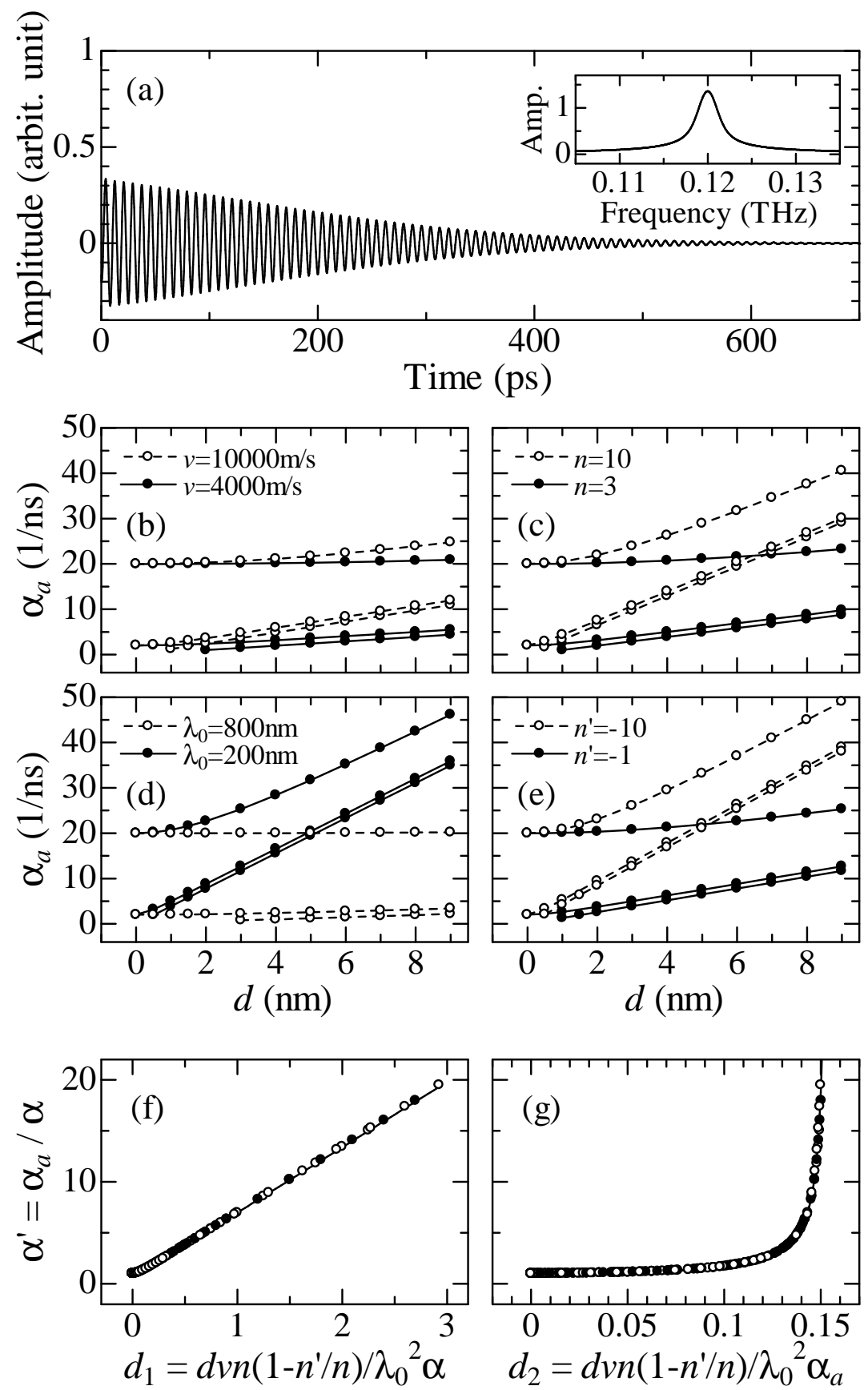

Fig. 4 